\title{
Does transition from standard to Retzius-sparing technique in robot-assisted radical prostatectomy affect the functional and oncological outcomes?
}

\author{
Hakan Anıl ${ }^{1}$, Kaan Karamık ${ }^{2}$, Ali Yıldız ${ }^{3}$, Murat Savaş ${ }^{4}$ \\ ${ }^{1}$ Department of Urology, Adana Seyhan State Hospital, Adana, Turkey; \\ ${ }^{2}$ Department of Urology, Antalya Korkuteli State Hospital, Antalya, Turkey; \\ ${ }^{3}$ Department of Urology, Okan University Hospital, Faculty of Medicine, Istanbul, Turkey; \\ ${ }^{4}$ Department of Urology, Antalya Memorial Hospital, Antalya, Turkey.
}

\begin{abstract}
Summary Objective: To appraise the outcomes on the Retzius-sparing robot-assisted radical prostatectomy (Rs-RARP) learning curve of a surgeon with previous experience of anterior (standard) RARP.

Materials and methods: The first 50 cases during the Rs-RARP learning curve (group 1) and 50 cases after the second 100 cases with the standard approach (group 2) were comprised in the study. Patients who used zero or one safety pads were considered continent. Erectile function recuperation was characterized as the competence to achieve penetrative intercourse without receiving any medication. All patients were reevaluated at two weeks, first, third, sixth, and $12^{\text {th }}$ months after surgery using IIEF-5, PSA level, and continence status.

Results: Immediate continence rates following catheter removal were $32 / 50$ (64\%) in Rs-RARP group and 26/50 (52\%) in $S$-RARP group $(p=0.224)$. The continence recovery rate was $48 / 50$ (96\%) in Rs-RARP group and 46/50 (92\%) in the S-RARP group at 12 months follow-up $(p=0.400)$. Total nerve-sparing surgery was enforced in 36/50 (72\%) patients for group 1 and $35 / 50(70 \%)$ patients for group 2. Potency recovery was $27 / 43$ (62.8\%) in Rs-RARP and 30/44 (68.2\%) for S-RARP at 12 months follow up $(p=0.597)$. Surgical margin positivity was detected in $6 / 50(12 \%)$ cases in the Rs-RARP group and in $4 / 50$ $(8 \%)$ cases in the S-RARP $(p=0.444)$.

Conclusions: Functional and oncological results are not negatively affected in the first 50 cases for a surgeon who is experienced in S-RARP before transition to the Rs-RARP method.
\end{abstract}

KEY WORDS: Learning curve; Radical prostatectomy; Retziussparing; Robotic surgery; Trifecta.

Submitted 28 August 2021; Accepted 19 September 2021

\section{INTRODUCTION}

Prostate cancer is the most frequently diagnosed type of cancer among men and is the second most common cause of cancer-related death in men (1). Radical prostatectomy is the most commonly offered treatment modality in eligible patient groups (2). Robot-assisted radical prostatectomy (RARP) has become popular in the last two decades. The method most widely adopted by urologists is standard-RARP with an anterior approach (3).

The main issue after RARP is preserving continence and erectile function, in addition to oncologic safety. Many techniques were reported to improve continence (4-6). Recently, a new method is the Retzius-sparing (Rs) RARP applied with the posterior approach, defined by Galfano et al. (7). The basic principle of this approach is to prevent interference with anatomical structures that provide continence in the anterior region. In this context, many surgeons may consider revising their method.

Additionally, it is controversial for a surgeon who has completed the learning curve with the standard approach to change their technique using the Rs-RARP approach with regard to functional and oncological results.

In this study aimed to appraise the functional and oncological outcomes during the Rs-RARP learning curve for a surgeon with previous experience of the S-RARP procedure.

\section{Materials AND METHOdS}

Patient selection and study design

After the local institutional review board approval, the RsRARP and S-RARP surgeries applied by a single surgeon with experience in robotic surgery between 01.01.2017 and 01.01.2019 were retrospectively evaluated from a prospectively-collected database. The first 50 cases during the Rs-RARP learning curve and 50 cases after the second 100 cases with the standard approach were enrolled in the study. Surgeon participated in live surgeries before performing Rs-RARP. In addition, in the first 10 cases, patients with low-to-moderate volume prostates $(\leq 60 \mathrm{cc})$ without a median lobe were preferred. Patients with previous prostate and abdominal surgery, incontinence, and presence of lymph node metastasis were excluded from the study. Fifty patients who met the inclusion criteria and underwent Rs-RARP were considered group 1, and 50 patients who underwent S-RARP were group 2.

\section{Evaluated variables}

Preoperative evaluation included age, Charlson Comorbidity Index score (CCI), prostate-specific antigen (PSA), International Index of Erectile Function-5 (IIEF-5) score, Gleason score at biopsy, clinical T stage, prostate volume, and D'Amico risk group. Furthermore, perioperative variables and pathologi-

No conflict of interest declared. 
cal features were noted: operative time, anastomosis duration, nerve-sparing status, intraoperative complications, estimated blood loss, presence of lymph node dissection, length of hospital stay, Foley catheter removal time, lymph node positiveness, surgical margin status, and pathological stage. Patients were followed for at 12 months for continence and potency recovery.

Immediate continence evaluation was performed within 1 week after catheter removal. Patients who used zero or one safety pads were considered continent. Erectile function recovery was evaluated in the first month, initially. Erectile function recuperation was characterized as the competence to achieve penetrative intercourse without the use of any medication. All patients were reevaluated at two weeks, first, third, sixth, and $12^{\text {th }}$ month after surgery using IIEF5, PSA level, and continence status.

\section{Surgical technique}

All robot-assisted radical prostatectomies were implemented by a single surgeon (M.S) who routinely performed over 100 RARPs per year. Our surgical principles were close to that defined by Galfano et al. (7). After the placement of 4 robot trocars and 1 assistant trocar, a 4 arms Da Vinci robot system (Intuitive surgical, LA, USA) was docked. The parietal peritoneum was incised horizontally at the anterior layer of the Douglas pouch. Seminal vesicle pedicles were identified and ligated by using Hem-o-Lok clips. Also, vas deferens were identified and incised.

The avascular zone was found by entering among the Denonvillier's fascia and the posterior prostatic fascia.

The intrafascial plan was maintained and the prostate apex was reached with dissection. The bladder neck was identified and incised. At this step, we don't use cardinal stitches to identify the bladder neck, unlike Galfano and colleagues (7). The anterior surface of the prostate was separated from the Santorini plexus. Apex separation was achieved and the urethra identified and cutted.

The prostate was placed in the endobag. The urethrabladder anastomosis was performed continuously with 3.0 v-lock sutures from the 12-o'clock position.

\section{Statistical analysis}

Variables were presented as mean \pm standard deviation, median and interquartile range $\left(25^{\text {th }}-75^{\text {th }}, I Q R\right)$, frequency, and percentage. Evaluation of categorical data was done with the chi-square or Fisher's exact test. The conformity of the data to the normal distribution was checked with the Shapiro-wilk test. Student's t-test or Mann-Whitney U test was used for continuous variables according to the distribution. The Kaplan Meier analysis was applied to determine erectile and continence recovery. A two-way repeated-measures ANOVA was used to compare preoperative and postoperative IIEF scores between surgical approaches. Statistical analysis was conducted with IBM SPSS Statistics for Windows, version 22.0 (IBM Corp., Armonk, NY).

A p-value $<0.05$ was considered as statistically significant.

\section{RESULTS}

Group 1 and group 2 comprised 50 patients with a median age of 63.5 (IQR 58-68.5) years, and 50 patients with a median age of 66 (IQR, 62-68.5) years, respectively.
Table 1.

Demoghraphics and preoperative features.

\begin{tabular}{|c|c|c|c|}
\hline Variable & Rs-RARP & S-RARP & $P$ value \\
\hline Median (IQR) age, years & $63.5(58-68.5)$ & $66(62-68.5)$ & $0.151^{T}$ \\
\hline Median (IQR) CCI score & $2(1-3)$ & $2(1-3)$ & $0.531^{T}$ \\
\hline$\overline{\text { Median (IQR) BMI, kg/m² }}$ & $27.2(24.3-28.7)$ & $28(25-29.7)$ & $0.223^{T}$ \\
\hline Median (IQR) PSA, ng/ml & $9.7(7.8-11.2)$ & $8.0(5-13.4)$ & $0.183^{T}$ \\
\hline $\begin{array}{l}\text { Gleason score at biopsy } n(\%) \\
\leq 6 \\
7 \\
8-10\end{array}$ & $\begin{array}{l}31(62) \\
13(26) \\
6(12)\end{array}$ & $\begin{array}{c}32(64) \\
14(28) \\
4(8)\end{array}$ & $0.797^{*}$ \\
\hline $\begin{array}{l}\text { Clinical T stage n }(\%) \\
\text { T1a-c } \\
\text { T2a-b } \\
\text { T2c }\end{array}$ & $\begin{array}{c}33(66) \\
15(30) \\
2(4)\end{array}$ & $\begin{array}{c}29(58) \\
19(38) \\
2(4)\end{array}$ & $0.695 *$ \\
\hline Mean ( \pm SD) prostate volume, $\mathrm{mL}$ & $60.1 \pm 24.2$ & $58.8 \pm 22.6$ & $0.782^{*}$ \\
\hline $\begin{array}{l}\text { D'Amico risk classification n (\%) } \\
\text { Low } \\
\text { Intermediate } \\
\text { High }\end{array}$ & $\begin{array}{l}23(46) \\
15(30) \\
12(24)\end{array}$ & $\begin{array}{l}28(56) \\
14(28) \\
8(16)\end{array}$ & $0.516^{*}$ \\
\hline
\end{tabular}

There were statistically insignificant differences among the groups regarding preoperative demographics and clinical characteristics (Table 1). The median operative time was 162.5 (IQR 137.5-210) mins and 150 (IQR 125-220) mins for groups 1 and 2 , respectively ( $\mathrm{p}=$ $0.865)$. Bilateral nerve-sparing surgery was performed in $36 / 50(72 \%)$ and $35 / 50(70 \%)$ patients for group 1 and group 2 , respectively. Intraoperatively, one patient had

Table 2.

Peroperative and postoperative results.

\begin{tabular}{|c|c|c|c|}
\hline Variable & Rs-RARP & S-RARP & $P$ value \\
\hline $\begin{array}{l}\text { Median (IQR) operative time, mins } \\
\end{array}$ & $162.5(137.5-210)$ & $150(125-220)$ & $0.865^{T}$ \\
\hline Nevve sparing, n (\%) & & & $0.843 *$ \\
\hline Bilateral & 36 (72) & $35(70)$ & \\
\hline Unilateral & $7(14)$ & $9(18)$ & \\
\hline None & 7 (14) & $6(12)$ & \\
\hline Lymph node dissection, $n(\%)$ & $21(42)$ & $19(38)$ & $0.683^{*}$ \\
\hline Median (IQR) anastomosis time, mins & $20(15-20)$ & $20(15-20)$ & $0.952^{T}$ \\
\hline $\begin{array}{l}\text { Median (IQR) blood loss, mL } \\
\end{array}$ & $150(100-200)$ & $100(65-150)$ & $0.120^{T}$ \\
\hline Median (IQR) hematocrit decrease & $4.2(0.7-1.7)$ & $3.9(0.7-1.6)$ & $0.668^{T}$ \\
\hline Median (IQR) discharged time, day & $3(2.7-3)$ & $3(2-3.5)$ & $0.588^{T}$ \\
\hline Median (IQR) catheter removal time, day & $8(7-9)$ & $8(7-9)$ & $0.431^{T}$ \\
\hline \multicolumn{4}{|l|}{$\mathrm{pT}, \mathrm{n}(\%)$} \\
\hline TO & 0 & $1(2)$ & \\
\hline T2 & $38(76)$ & 36 (72) & \\
\hline ТЗа & $7(14)$ & $8(16)$ & \\
\hline T3b-4 & $5(10)$ & $5(10)$ & \\
\hline \multicolumn{4}{|l|}{$\overline{\mathrm{pN}, \mathrm{n}(\%)}$} \\
\hline NO & $20(40)$ & $18(36)$ & \\
\hline $\mathrm{N}^{+}$ & $1(2)$ & $1(2)$ & \\
\hline Positive surgical margin, $n(\%)$ & & & $0.444^{*}$ \\
\hline Yes & $6(12)$ & $4(8)$ & \\
\hline No & $44(88)$ & $46(92)$ & \\
\hline
\end{tabular}


complete ureteral injury in the Rs-RARP group. One patient in the S-RARP group had external iliac vein injury during lymph node dissection. Both complications were managed intracorporeally. As a result of the histopathological examination of radical specimens, surgical margin positivity was detected in $6 / 50$ (12\%) cases in the RsRARP group and in $4 / 50(8 \%)$ cases in the S-RARP ( $p=$ 0.444). Intra-postoperative clinical and pathological outcomes are summarized in Table 2.

The median catheter discharge time was 8 days (IQR 7-9) in group 1 and 8 (IQR 7-9) days for group 2, respectively $(\mathrm{p}=0.431)$. Immediate continence rates following catheter removal were 32/50 (64\%) in the Rs-RARP group and $26 / 50(52 \%)$ in the S-RARP group $(\mathrm{p}=0.224)$.

The continence recovery rate was $48 / 50$ (96\%) in the RsRARP group and 46/50 (92\%) in the S-RARP group at 12 month follow-up $(p=0.400)$. Comparison of both groups according to continence recovery using KaplanMeier methods is shown in Figure 1A. Eighty-seven patients, 43 in group 1 and 44 in group 2, who achieved preoperative sexual intercourse were included in the erectile function evaluation. The mean baseline IIEF-5 score was $21.6 \pm 2.5$ and $20.7 \pm 2.6$ in group 1 and 2 , respectively ( $p=0.143)$. No statistically significant difference was found between the surgical approach in terms of IIEF score reductions at 12 -month follow-up $(p=0.260)$ (Figure 1B). Potency recovery of patients who underwent bilateral or unilateral nerve-sparing surgery was $27 / 43$ (62.8\%) in group 1 and 30/44 (68.2\%) in group 2 at 12 months follow up. This difference was not statistically significant $(p=0.597)$. Figure $1 C$ shows the KaplanMeier curve for potency recovery at 12-months follow-up $(\mathrm{p}=0.719)$. Biochemical recurrence was observed in $7 / 50$ (14\%) patients in group 1, and 6/50 (12\%) patients in group 2 at median 33 (IQR 28-40) months follow-up ( $\mathrm{p}=$ 0.766 ). Biochemical recurrence-free survival analysis according to surgical approaches is shown in Figure 1D.

\section{Discussion}

One-third of men with localized prostate cancer undergo radical prostatectomy (8). Incontinence is an adverse effect faced by patients after radical prostatectomy. Rs-RARP recently gained popularity with early continence results. In this article, we questioned the effect of the Rs-RARP learning curve on functional and oncological results for a sur-

Figure 1.

Comparison of functional and oncological outcomes between groups.

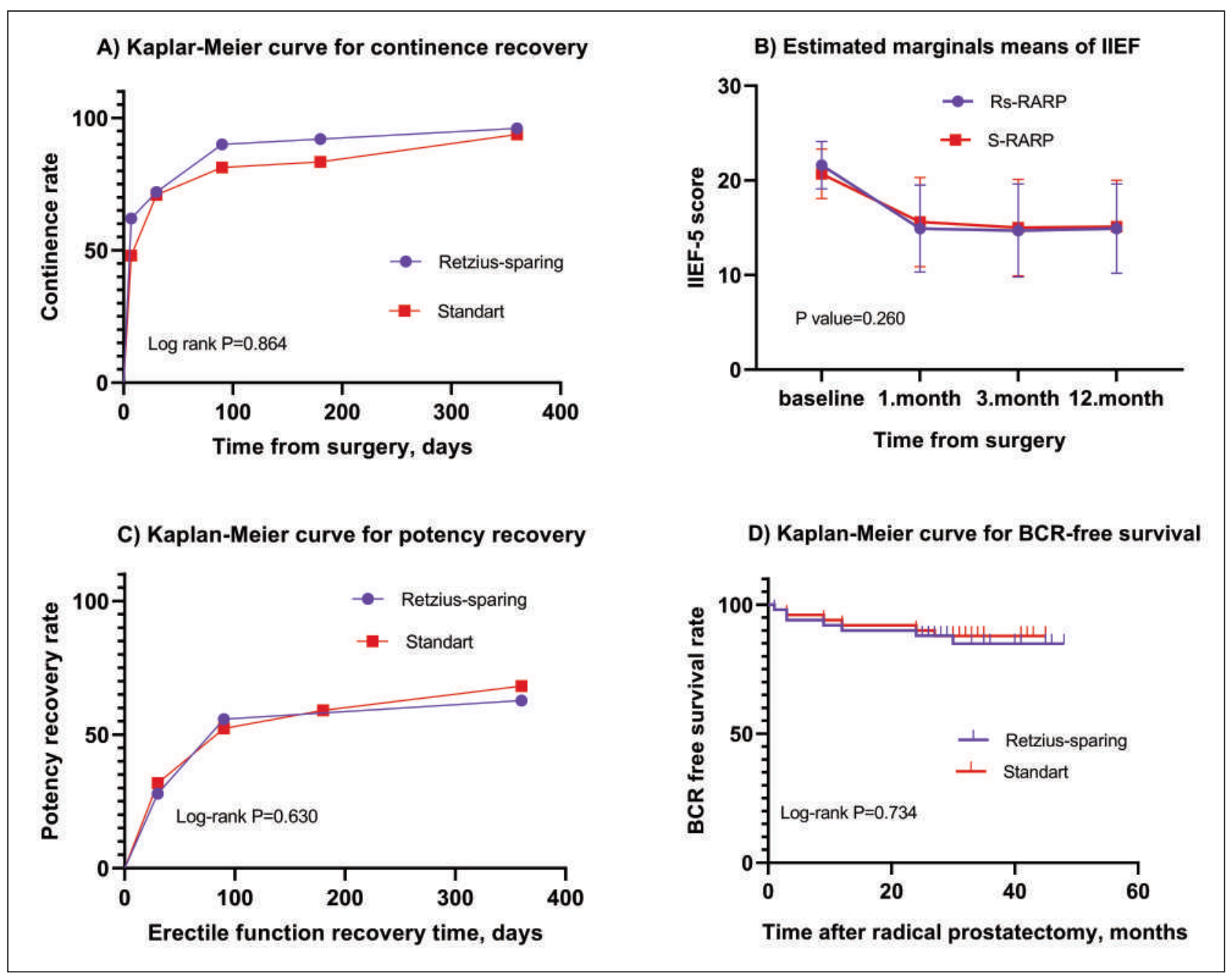


geon who was experienced in S-RARP before. This study provided that the Rs-RARP learning curve period is not adversely affected by either functional or oncologic results. The number of cases required to achieve competence in radical prostatectomy is uncertain. Also, there is no objective parameter to be used in defining the learning curve. A study based on complications showed that the complications were significantly reduced after 150 cases (9). In another study examining the learning curve for robotic radical prostatectomy by an experienced surgeon with the open approach, they reported that self-confidence and comfort similar to open surgery were achieved after performing 250 robotic surgeries (10). In a recent study, Islamoglu et al. reported that the surgeon experienced in laparoscopy and open radical prostatectomy should have experience of at least 50 cases to achieve the optimal surgical time. However, they found that the learning curve did not affect positive surgical margin (11). Another study reported the requirement of 90 cases after intensive structured modular training to achieve optimal perioperative and functional outcomes (12).

In this context, we think that the learning curve for RsRARP is person-based and will vary depending on the surgeon's previous skills in laparoscopy and S-RARP.

In 2010, Galfano et al. defined Rs-RARP for the first time in their study, which they defined as the Bocciardi approach (7). The theory of this approach is that it has a positive effect on continence and erectile function by protecting structures such as the Santorini plexus, pudendal artery, and pubourethral ligament. Recent studies show that Rs-RARP is advantageous especially in terms of immediate continence (13). In their series of 200 cases, Galfano et al. reported a continence rate of $90 \%$ at 1 week and $96 \%$ at the end of 1 year (14). In a randomized controlled study comparing Rs-RARP with S-RARP, continence rates at 1 week were $71 \%$ versus $48 \%$ in favor of Rs-RARP ( $p=0.01$ ) (2). In a series of 256 cases involving surgeons on the learning curve, the immediate continence rate was reported as $82 \%$ and $90 \%$ at the end of 12 months (15). On the other hand, it was emphasized that the high continence rate seen in the early period with the Rs-RARP approach is similar to S-RARP after 12 months (12). In our study, although the continence rates in the first week were higher in the Rs-RARP group compared to the S-RARP group, no statistical difference was found. At the end of the first year, results were excellent in both groups.

Sexual potency evaluation was performed in patients with preoperative penetrative sexual intercourse and patients undergoing bilateral or unilateral nerve-sparing surgery. At the end of 12 months, we found a potency rate of $62.8 \%$ in the Rs-RARP group and $68.2 \%$ in the S-RARP group. Olivero and colleagues reported a sexual potency rate of $80.4 \%$ in their study with surgeons on the RsRARP learning curve. However, they included young patients with full-nerve-sparing procedures in the analysis in this study (15). In a randomized prospective study including $3^{\text {rd }}$-month penetrative intercourse rates for 30 anterior and 30 posterior approaches by Mennon et al. rates were reported as $36.7 \%$ in the anterior group and $43.7 \%$ in the posterior group. They emphasized that at the end of the $12^{\text {th }}$ month erection sufficient for penetrative intercourse increased to $69.2 \%$ in the anterior group and $89 \%$ in the posterior group (16). In our study, the rate of erectile function was found to be lower in the RsRARP group compared to the literature. This may be because we included patients from all age groups and patients who underwent unilateral nerve-sparing surgery. Positive surgical margin is a valuable data for evaluating oncological outcomes after radical prostatectomy. Galfano et al. found a PSM rate of $22 \%$ in the first 100 cases in their learning curve and 9\% in the second 100 patients (14). Sayyid and colleagues reported that PSM after RsRARP and S-RARP were similar in accordance to pT stage subgroups (for pT2 stage: $16.7 \%$ PSM in Rs-RARP vs $13.7 \%$ PSM in S-RARP, $\mathrm{p}=0.54$; for $\mathrm{pT} 3$ stage: $47.1 \%$ and $47.8 \%$, respectively, $\mathrm{p}=0.95)$ (17). In another study comparing Rs-RARP and S-RARP, the rate of PSM was 10\% in the S-RARP group and $28.2 \%$ in the Rs-RARP group, and this difference was statistically significant $(p=0.05)$. However, when $\mathrm{pT}$ stage subgroups were compared, no statistical significance was found (18). Also, many studies comparing Rs-RARP and S-RARP reported similar PSM rate and biochemical recurrence-free survival (8). In our study, in accordance with the literature, there was no significant difference in PSM rate between the groups.

Our study has some limitations. First bias may be due to the retrospective design. Second, the study has a relatively small sample size. On the other hand, our data were collected prospectively and all surgical procedures were performed by a single surgeon.

\section{Conclusions}

This study reported the outcomes for cases on the Rs-RARP learning curve of a surgeon with previous experience of S-RARP. According to the data in our study, when a surgeon who is experienced in S-RARP switches to the RsRARP method, functional and oncological results are not negatively affected in the first 50 cases. Although immediate continence was found to be lower than the literature in our study, the results are excellent at the end of 12 months. Surgeons previously experienced in the S-RARP approach can safely move to the Rs-RARP approach.

\section{REFERENCES}

1. Siegel RL, Miller KD, Jemal A. Cancer statistics, 2018. CA Cancer J Clin. 2018; 68:7-30.

2. Dalela D, Jeong W, Prasad MA, et al. A pragmatic randomized controlled trial examining the impact of the Retzius-sparing approach on early urinary continence recovery after robot-assisted radical prostatectomy. Eur Urol. 2017; 72:677-685.

3. Galfano A, Secco S, Dell'Oglio P, et al. Retzius-sparing robotassisted radical prostatectomy: early learning curve experience in three continents. BJU Int. 2021; 127:412-417.

4. Patel VR, Coelho RF, Palmer KJ, Rocco B. Periurethral suspension stitch during robot-assisted laparoscopic radical prostatectomy: description of the technique and continence outcomes. Eur Urol. 2009; $56: 472-8$.

5. Ma X, Tang K, Yang C, et al. Bladder neck preservation improves time to continence after radical prostatectomy: a systematic review and meta-analysis. Oncotarget. 2016; 7:67463-75. 
6. Kojima Y, Takahashi N, Haga N, et al. Urinary incontinence after robot-assisted radical prostatectomy: pathophysiology and intraoperative techniques to improve surgical outcome. Int J Urol. 2013; 20:1052-63.

7. Galfano A, Ascione A, Grimaldi S, et al. A new anatomic approach for robot-assisted laparoscopic prostatectomy: a feasibility study for completely intrafascial surgery. Eur Urol. 2010; 58:457-61.

8. Davis M, Egan J, Marhamati S, et al. Retzius-Sparing RobotAssisted Robotic Prostatectomy: Past, Present, and Future. Urol Clin North Am. 2021; 48:11-23.

9. Ou Y-C, Yang C-R, Wang J, et al. The learning curve for reducing complications of robotic-assisted laparoscopic radical prostatectomy by a single surgeon: complications of RALP. BJU Int. 2011; 108:420-5.

10. Herrell SD, Smith JA Jr. Robotic-assisted laparoscopic prostatectomy: what is the learning curve? Urology. 2005; 66 (5 Suppl):105-7.

11. Islamoglu E, Karamik K, Ozsoy C, et al. The learning curve does not affect positive surgical margin status in robot-assisted laparoscopic prostatectomy. Urol J. 2018; 15:333-338.

12. Schiavina R, Borghesi $M$, Dababneh $H$, et al. The impact of a structured intensive modular training in the learning curve of robot assisted radical prostatectomy. Arch Ital Urol Androl. 2018; 90:1-7.
13. Phukan C, Mclean A, Nambiar A, et al. Retzius-sparing robotic assisted radical prostatectomy vs. conventional robotic assisted radical prostatectomy: a systematic review and meta-analysis. World J Urol. 2020; 38:1123-1134.

14. Galfano A, Di Trapani D, Sozzi F, et al. Beyond the learning curve of the Retzius-sparing approach for robot-assisted laparoscopic radical prostatectomy: oncologic and functional results of the first 200 patients with $\geq 1$ year of follow-up. Eur Urol. 2013; 64:974-80.

15. Olivero A, Galfano A, Piccinelli M, et al. Retzius-sparing robotic radical prostatectomy for surgeons in the learning curve: a propensity score-matching analysis. Eur Urol Focus. 2021; 7:772-778.

16. Menon $M$, Dalela $D$, Jamil $M$, et al. Functional recovery, oncologic outcomes and postoperative complications after robot-assisted radical prostatectomy: an evidence-based analysis comparing the Retzius-sparing and standard approaches. J Urol. 2018; 199:12101217

17. Sayyid RK, Simpson WG, Lu C, et al. Retzius-sparing roboticassisted laparoscopic radical prostatectomy: a safe surgical technique with superior continence outcomes. J Endourol. 2017; 31:1244-1250.

18. Asimakopoulos AD, Topazio L, De Angelis M, et al. Retzius-sparing versus standard robot-assisted radical prostatectomy: a prospective randomized comparison on immediate continence rates. Surg Endosc. 2019; 33:2187-2196.

\section{Correspondence}

Hakan Anil, MD (Corresponding Author)

dr.hakananil@gmail.com

Department of Urology, Adana Seyhan State Hospital, Adana (Turkey)

Kaan Karamik, MD

Department of Urology, Antalya Korkuteli State Hospital, Antalya (Turkey)

Ali Yildız, Asst. Prof., MD

Department of Urology, Okan University Hospital, Faculty of Medicine,

Istanbul (Turkey)

Murat Savas, Prof. Dr., MD

Department of Urology, Antalya Memorial Hospital, Antalya (Turkey) 